\title{
Observation of coherent terahertz edge radiation from compressed electron beams
}

\author{
G. Andonian, A. Cook, M. Dunning, E. Hemsing, G. Marcus, A. Murokh, S. Reiche, D. Schiller, and J. B. Rosenzweig \\ Department of Physics and Astronomy, University of California, Los Angeles, Los Angeles, California 90095, USA
}

\author{
M. Babzien, K. Kusche, and V. Yakimenko \\ Accelerator Test Facility, Brookhaven National Laboratory, Upton, New York 11973, USA
}

(Received 13 November 2006; published 3 March 2009)

\begin{abstract}
Coherent radiation emitted from a compressed electron bunch as it traverses the sharp edge regions of a magnetic chicane has been investigated at the Brookhaven National Laboratory Accelerator Test Facility. Electron beam measurements using coherent transition radiation interferometry indicate a $100 \mathrm{fs}$ rms bunch accompanied by distinct distortions in energy spectrum due to strong self-fields. These self-fields are manifested in emitted high power THz radiation, which displays signatures of the phenomenon known as coherent edge radiation. Radiation characterization studies undertaken include spectral analysis, farfield intensity distribution, polarization, and dependence on the electron bunch length. The observed aspects of the beam and radiation allow detailed comparisons with start-to-end simulations.
\end{abstract}

DOI: 10.1103/PhysRevSTAB.12.030701

PACS numbers: 41.75.Ht, 41.60.Ap, 41.60.Cr, 42.30.Rx

Novel types of coherent electromagnetic radiation sources based on electron beams, extending in spectrum from $\mathrm{THz}$ to $\mathrm{x}$ rays, are presently under intensive development in the scientific community. For example, the x-ray free-electron laser (FEL) is a light source that will allow investigation of physical systems on the time and length scales of atomic motion (100 fs, $1 \AA$ ) [1], paving the way for revolutionary advances in biological and condensed matter sciences [2]. Such sources are based on emission from high brightness (high phase space density), relativistic electron beams undergoing magnetic bending forces. Specifically, x-ray FELs require short electron pulses simultaneously having high charge and low energy spread, with small transverse normalized emittance, $\epsilon_{n}$.

The requirements for achieving high-gain lasing in an $\mathrm{x}$ ray FEL illustrate this basis, as one typically must enhance the beam current, $I_{b}$, from $100 \mathrm{~A}$ at the source to several $\mathrm{kA}$, while maintaining $\epsilon_{n}$ on the order of $1 \mathrm{mmmrad}$. Obtaining such beams entails pulse compression, commonly implemented by deploying a magnetic chicane array downstream of a radio-frequency linear accelerator ( $\mathrm{rf}$ linac). The linac is operated off the phase of maximum acceleration, imparting a time-energy correlation, or energy chirp, onto the beam. This chirp is then removed by the energy dependence of transport time through the chicane, thus yielding a beam shorter in time duration.

The compression process provokes strong selfinteractions in the beam, which manifest themselves in several ways. At lower energies ( $\leq 50 \mathrm{MeV}$ ), collective interactions are dominated by velocity field-derived effects, which strongly distort the transverse phase space [3]. At higher energies, acceleration fields are stronger; these give rise to both coherent synchrotron radiation (CSR) and an associated strong energy modulation of the beam [4]. This modulation may bring about a microbunch- ing instability [5], which has strong similarities to the FEL instability [6]. Such collective effects degrade the beam's longitudinal and transverse phase space during compression [7], and may severely impact the utility of a beam as an x-ray FEL lasing medium.

Previous studies of picosecond pulses in bends have observed CSR [8] and associated beam phase space distortions [3,9]. An interesting attribute of coherent radiation from bending electron beams is short wavelength $(\lambda<$ $1 \mathrm{~mm}$ ) [10] coherent edge radiation (CER). Edge radiation [11], which is created where the electrons enter and exit magnetic dipole field regions, has notable differences with respect to synchrotron radiation. In many respects it is similar to transition radiation, having a polarization component out of the bend plane, a hollow transverse spatial distribution, and a low-frequency spectral intensity nearly independent of frequency. Further, the frequency scaling of the coherent energy emitted (with $\lambda / 2 \pi$ longer than the rms bunch length, $\sigma_{z}$ ) via CER in an edge region is larger than that of CSR in the same relevant solid angle.

There are many proposed practical applications which exploit these features of coherent edge radiation. For example, CER is attractive as a basis for bunch length diagnosis. In particular, in future light sources, a nondestructive bunch profile monitor may be developed by exploiting the CER observed from the chicane magnet compressors employed to achieve the necessary high peak current. Interferometric analysis of the produced CER would yield direct information about the beam temporal profile. CER is also of interest as a unique, high power source of coherent $\mathrm{THz}$ radiation, which has a myriad of scientific, industrial, and biomedical applications, such as organic molecule imaging [8]. Since CER production is of interest for, and is already finding [12], widespread applications, it is worth a more concerted effort to study the principles of this 
radiation and its properties both analytically and experimentally.

In this paper, we report on measurements of the coherent radiation from electron bunches compressed to the levels expected in x-ray FELs, $100-150$ fs. This radiation is collected from the edge regions of magnets in the UCLA chicane at the Brookhaven National Laboratory Accelerator Test Facility (BNL ATF). Aspects of the radiation, such as polarization, wavelength spectrum, and far-field angular intensity are examined. Indeed, signature traits of CER are identified in the data obtained. These observations are correlated with beam pulse length and momentum spectral measurements. Both beam and radiation measurements are compared to detailed start-to-end simulations, which produce many, but not all, of the attributes found in the experiment.

The UCLA chicane is a four-bend, close-packed magnet array with a nominal vertical bend angle of $20^{\circ}$ and a radius of curvature of $1.2 \mathrm{~m}$. This corresponds to a $1.7 \mathrm{kG}$ bend field at the $61 \mathrm{MeV}$ beam energy used. The component dipole magnets are rectangular in the interior (second and third magnets), yet have final input and output edge angles $\left(20^{\circ}\right)$ that are horizontally defocusing, in order to avoid bringing the beam to a tight focus while bending, and exacerbating density dependent collective effects.

The linear transport coefficient, $R_{56}=\frac{\partial \xi}{\partial(\delta p / p)}$, relates the change in an electron's longitudinal position within the bunch, $\xi=z-c t$, to its relative momentum deviation from the design particle, $\delta p / p$ [13]. For the given parameters of the UCLA chicane, $R_{56}=7.3 \mathrm{~cm}$. For complete compression, the optimum energy chirp on the beam is imparted by running at an $\mathrm{rf}$ linac phase $11^{\circ}$ forward of the minimum momentum spread condition.

Start-to-end simulations were performed using UCLA PARMELA [14] for photoelectron beam generation and acceleration in the rf gun and linac, and ELEGANT [15] for beam transport dynamics, including chicane compression modeling. Additional beam simulations, including beam self-interactions and radiation production, were performed with TREDI [16]. Simulations with UCLA PARMELA and ELEGANT indicate that nominal experimental conditions (bunch charge, $Q=300 \mathrm{pC}$; rms bunch length, $\sigma_{z}=$ $310 \mu \mathrm{m}$ ) yield a compressed bunch length as short as $\sigma_{z}=23 \mu \mathrm{m} \mathrm{rms}$. In this case, ELEGANT predicts that the rms bend-plane emittance grows in value from $\epsilon_{n, y}=$ $2.0 \mathrm{~mm} \mathrm{mrad}$ to $\epsilon_{n, y}=5.75 \mathrm{~mm} \mathrm{mrad}$, an increase experimentally verified in Ref. [17].

The chicane vacuum vessel features a port that allows for transmission of the radiation emitted near the line of the beam trajectory as it traverses the exit of the third magnet and entrance of the final magnet. The radiation is transmitted through a synthetic z-cut quartz window located at the exit of the port. After collimation with a Picarin lens, the radiation is propagated over $7 \mathrm{~m}$ of transport, where it is either: focused directly into cryogenic Si bolometer for total energy measurement; autocorrelated to yield a frequency spectrum; or directed through a movable smallaperture iris to generate an angular distribution. The measurements are performed in tandem with polarization variation using a rotatable wire-grid polarizer.

These radiation diagnostics are complemented by beam diagnostics downstream of the chicane. In the longitudinal phase plane, the momentum spectrum is measured in a magnetic spectrometer, equipped with collimating slits for precision measurement. The bunch time profile is determined through the autocorrelation of the coherent transition radiation (CTR) signal emitted from an insertable foil downstream of the chicane. The beam transverse phase space has been characterized separately by tomographic techniques, as reported in Ref. [17].

Compressed beams at moderate energy display a feature of longitudinal phase space breakup [9], manifested in the dipole spectrometer as splitting of the momentum spectrum (Fig. 1). The observed correlation between momentum spectrum bifurcation and beam compression allows for the use of this splitting as a diagnostic for initial optimization of the compression process; the chicane magnet values are varied until the characteristic beam splitting is observed. To calculate this spectrum we use TREDI simulations which, while providing a detailed model of the beam self-interactions, do not display the severity of the structure shown in measurements. This type of behavior has been reported in Ref. [18]; it may also be partly attributable to possible nonuniformities in the temporal profile of the photocathode drive laser [19]. The observed structure in the momentum spectrum is also correlated to a similar, yet gentler, splitting in the transverse phase space observed using tomographic techniques [17]. Tuning the beam requires strict monitoring of residual dispersion; the dispersion is mitigated by adjustments to magnet setting while observing beam centroid behavior, for varying $\mathrm{rf}$ phase conditions, at phosphor screens located before and after the chicane. The beam trajectory is set to simultaneously allow for optimum compression and maximal coherent radiation emitted into the collection/transport line.

The compressed beam time profile is determined by autocorrelating the CTR signal using a Michelson-type interferometer with Golay cell detectors. CTR is produced by the electron beam striking a $45^{\circ}$ aluminum target just downstream of the chicane compressor; radiation is transmitted through a synthetic z-cut quartz vacuum window into the interferometer. The interferometer, optimized in the range $\lambda=15-1000 \mu \mathrm{m}$, has a translatable mirror along one leg with $1 \mu \mathrm{m}$ spatial resolution [20]. The autocorrelation trace produced by the interferometer is analyzed using a Kramers-Kronig approach to phase reconstruction [21], and a time-domain profile is generated (Fig. 2). For the case of optimum compression shown in Fig. 2, the beam has a well-defined current peak greater 

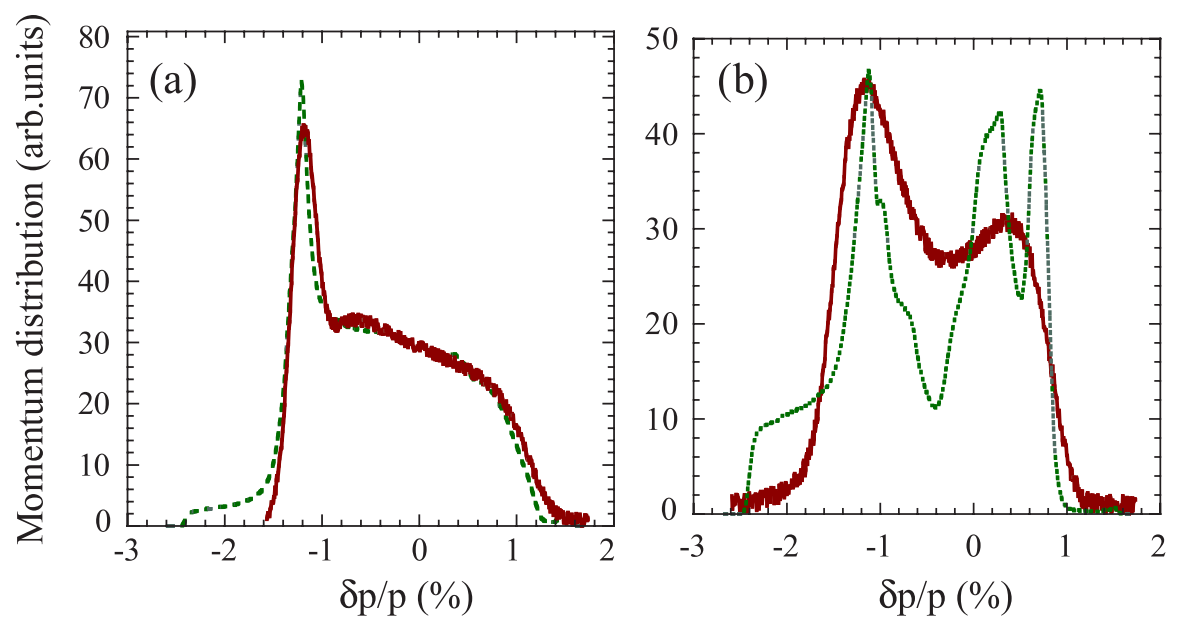

FIG. 1. (Color) (a) Typical electron beam momentum spectrum in the uncompressed phase: measured (dashed blue) and in TREDI simulation (solid red). (b) Typical momentum spectrum of an optimally compressed beam: measured (dashed blue) and in simulation (solid red).

than $500 \mathrm{~A}, 370 \mathrm{fs}$ FWHM (140 fs rms). The value for peak current is consistent with simulations while further iterations of this analysis have demonstrated peak currents as short as $100 \mathrm{fs}$ rms, consistent with bunch lengths observed by other experiments at the BNL ATF [22].

The beam compression process is achieved by running the linac phase forward of accelerating voltage crest. Running the linac at phases additionally forward of crest produces a scenario of over-compression of the electron beam; in this case, momentum spectrum modulation occurs earlier in the chicane compared to the optimal on-crest condition. The over-compressed beam experiences a larger fraction of the chicane $R_{56}$ and thus the momentum spectrum modulation, in combination with the larger longitudinal motion, yields a strong bifurcation in the beam temporal profile.

For the purposes of this experiment, the collected chicane radiation was scanned over a range of compression states (by varying the phase in the rf linac as described above), and revealed a strong correlation between the peak power and bunch length. The maximum chicane radiation was found at the minimum bunch length, as deduced from CTR analysis, indicating the dependence of coherent radiation production on achieving a short beam.

In order to predict the attributes of the bend-induced radiation at the detection plane, a new simulation tool was developed. This code, named QUINDI [23], employs macroparticles with initial conditions obtained from the output of PARMELA simulations. After generating particle spatial, velocity, and acceleration histories in the chicane, this trajectory information is used to calculate the radiation fields at the detector plane employing the LenardWiechert formalism. Further analysis of this data yields the frequency spectrum and angular distribution of the emitted radiation.

To measure the frequency spectrum, the collected radiation was autocorrelated using the interferometer with a cryogenically cooled Si bolometer detector. This measurement was performed for both CTR (Fig. 3) and CER emission (Fig. 4). The Fourier transform of the resulting
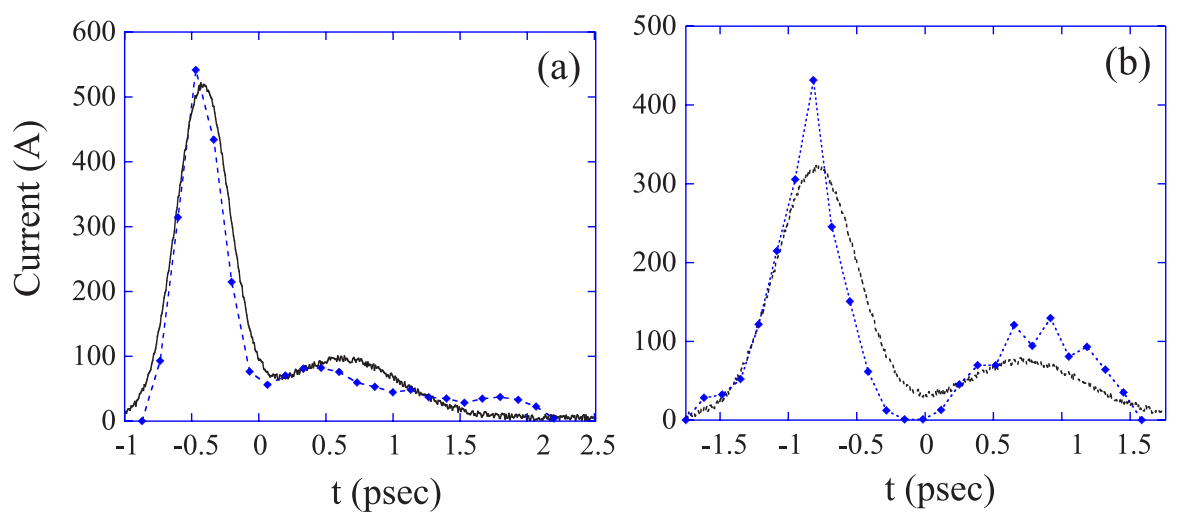

FIG. 2. (Color) Longitudinal beam density profiles reconstructed from Kramers-Kronig analysis of CTR autocorrelation, for cases of optimum compression (a) and over-compression (b): measured data (diamonds with dashed line) and simulation (solid line). 


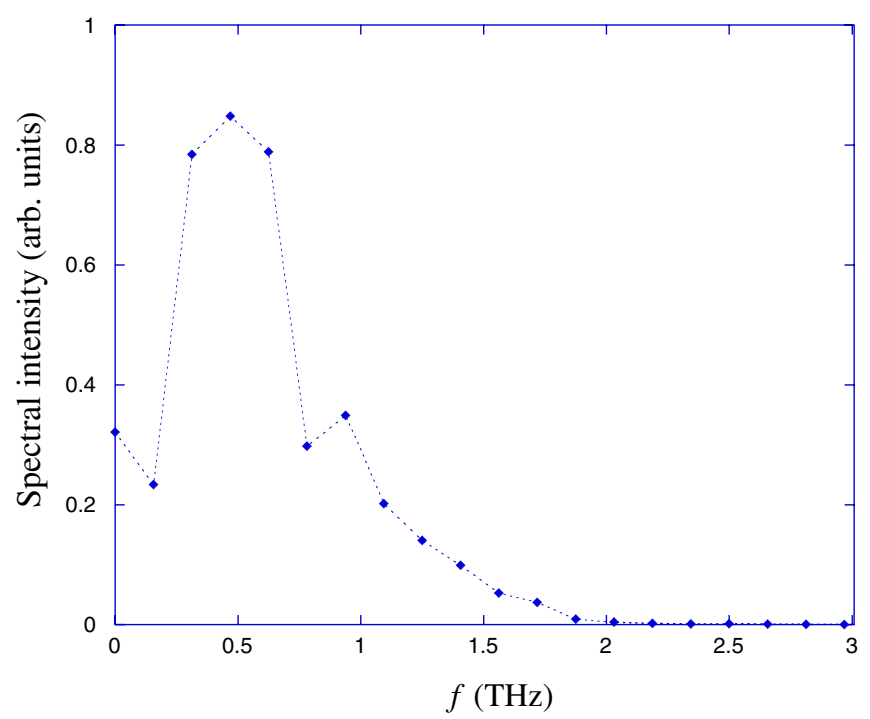

FIG. 3. (Color) Example of experimental spectrum generated from CTR interferometry data.

autocorrelation, although temporally dispersed during propagation, returns the reproducible radiation intensity spectrum, inclusive of absorption effects in transport. The resulting spectrum is displayed in Fig. 4, along with QUINDI predictions and comparison to spectra generated from CTR interferometry. The code ZEMAX was incorporated into the start-to-end simulations to model the transport and apertures from the point of emission to the detector plane. The overall frequency domain and peak near $0.5 \mathrm{THz}$ is con-

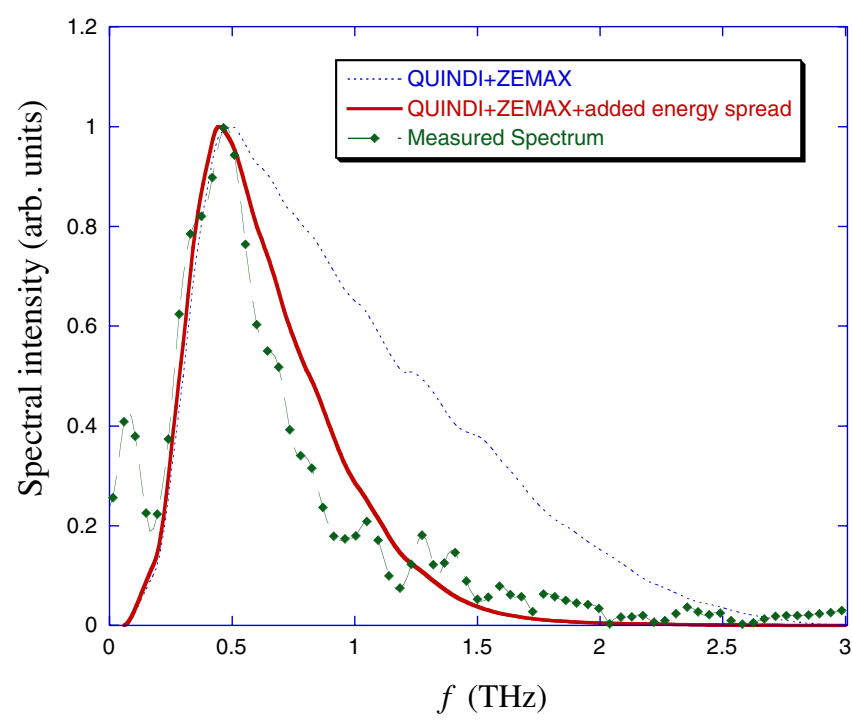

FIG. 4. (Color) Experimental spectra generated from the chicane radiation (green dots and dashes). Simulated spectra from QUINDI output for a cold beam (blue dotted curve) inclusive of ZEMAX transport corrections and for a beam with artificially added energy spread (red solid curve). The uncorrelated energy spread used for the fitting in the latter spectrum is $0.36 \%$ FWHM. sistent for both CTR and CER measurements and coincide with the results obtained from simulations using specific beam models. In the experiment, the CSR/CER induced energy changes serve to prevent formation of very short temporal structures in the beam. As the details of this interaction are outside of the resolving capability of the present computational model, uncorrelated (slice) energy spread was purposefully added into the beam distribution to artificially limit the formation of these sub-100 fsec features. Indeed, this uncorrelated energy spread in simulation was used as a fitting parameter for the measured spectral data. The value which provided the best fit to data was an energy spread of $0.36 \%$ FWHM. The comparison at high frequencies is markedly improved by this approach, which limits the peak simulated beam current to $370 \mathrm{~A}$ in QUINDI. This value is within the range of relevant TREDI simulation results, albeit a bit smaller than the experimentally deduced currents, as can be noted from Fig. 2. Diffraction losses at large wavelengths, as well as vacuum chamber shielding effects on radiation production, also yield artifacts in the measured spectrum omitted by simulations.

The overall radiation polarization is a telling measure in distinguishing between CER and CSR. CER has near equal $\sigma$ and $\pi$ polarization in the limit of zero-length magnetic field transition, while CSR is dominated by $\sigma$ polarization [24]. The polarization is determined with a wire-grid polarizer, mounted at the end of the transport line, rotated in $15^{\circ}$ increments. Figure 5 shows the measured intensity, normalized to its maximum value, as a function of the polarizer angle. The sinusoidal dependence on the polarizer angle is consistent with that expected from nearly linearly polarized CSR, while the vertical offset of the curve minima from zero indicates the presence of an orthogonal ( $\pi$-polarization) component, a distinct trademark of CER. QUINDI predictions are consistent with the behavior of the observed data (Fig. 5).

Further investigation of the radiation characteristics includes a detailed study of the transverse far-field angular

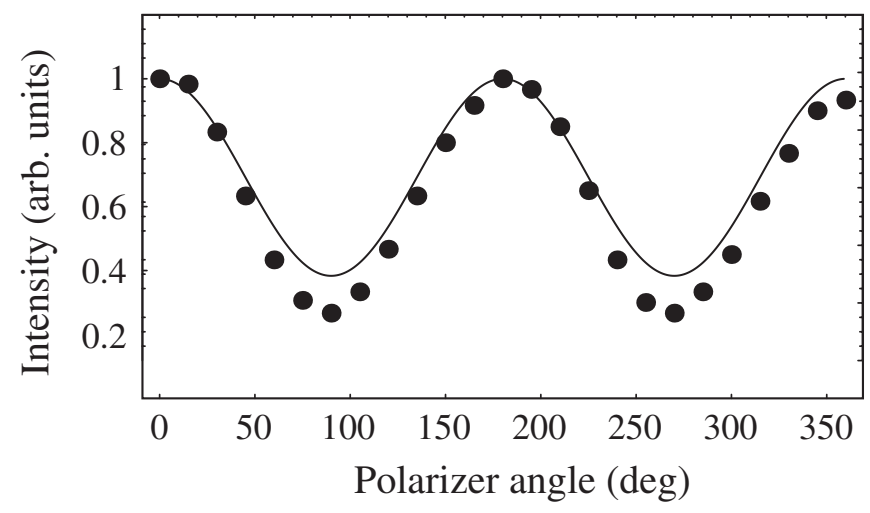

FIG. 5. The normalized polarization intensity of the measured chicane radiation (dots) exhibits the polarization predicted from QUINDI simulations (solid curve). 

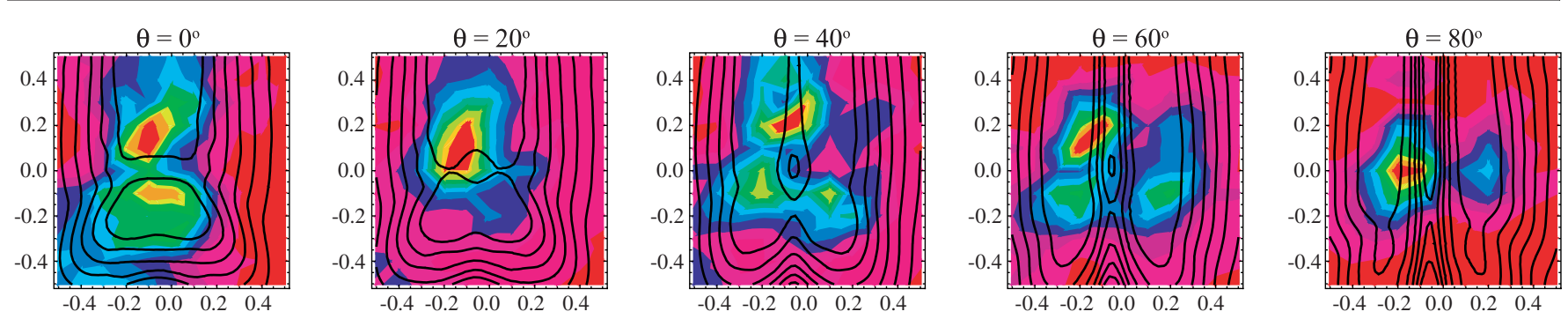

FIG. 6. (Color) Interpolated far-field radiation intensity distributions for $20^{\circ}$ polarizer angle increments represented in false color, with results from QUINDI simulations (contours) overlayed. The abscissa and ordinate have units of $\mathrm{mm}$.

intensity distribution, with an examination of its dependence on polarization. The far-field angular distribution after transport was measured by scanning a small iris (3 mm diameter) in a rectangular array of discrete points $(\Delta d=3.8 \mathrm{~mm})$ across the transport line exit. In order to best compare QUINDI predictions to data, the simulation results were scaled to account for the mitigated water absorption and other transmission efficiencies in the collection/transport line. The measured radiation profiles, for varying polarizations, of Fig. 6 (false color intensity plots) are superimposed on the isointensity contours predicted by QUINDI simulations (black contour lines). There is a modest agreement in the degree of compactness of the distributions, as well as in the trends of angular spreading and the number and direction of observed intensity lobes.

Some experimentally noted features of the angular distribution are not accounted for in the QUINDI model. Angular distribution patterns for short wavelength radiation are strongly affected by the details of the electron beam spatial distribution [25]. However, in our approach, the strong distortions due to collective effects, which are difficult to model numerically due to the large number of simulation particles required $[3,17]$, are not reproduced in detail. At present higher resolution QUINDI simulations using massively parallel computing techniques are being attempted in order to study these effects. On the opposite end of the radiation spectrum, our model does not account for vacuum vessel shielding effects. In this regard, we note that alternative approaches to modeling radiation fields including boundary effects have been developed but are not capable of calculating beam self-forces [26].

In conclusion, distinct signatures of coherent edge radiation from an ultrashort electron beam in a chicane were experimentally studied and the results benchmarked to beam measurements and simulations. The observed signatures, including polarization, wavelength spectrum, and angular distribution associated with the emitted radiation, are consistent with theoretical models, elucidated by simulation. Forthcoming improvements in the experimental and computational understanding of CER production, including simulation of relevant beam dynamics, are essential, especially from the standpoint of use of CER obtained from chicanes as a coherent $\mathrm{THz}$ source [8] or as a beam diagnostic [25].
Indeed, as next-generation light sources employ bunch compression schemes such as chicane magnet compressors to achieve the necessary peak beam currents for advanced experiments, a nonintercepting beam diagnostic, exploiting the CER radiation produced, would impart critical beam information depending on the numerical reconstruction method used. The possibility of monitoring the beam temporal profile during compression, as well as the stability of the compression process, would allow for real-time feedback systems that would improve overall beam quality for various experimental applications. The initial commissioning of a CER based bunch length diagnostic has been reported in Ref. [27]. The further development of such a diagnostic necessitates a careful and detailed characterization of the properties of the emitted radiation as presented in this paper.

\section{ACKNOWLEDGMENTS}

This work was performed under partial support of Department of Energy Contract No. DE-FG-98ER45693, and Office of Naval Research Contract No. N000140210911.

[1] C. Pellegrini and J. Stohr, Nucl. Instrum. Methods Phys. Res., Sect. A 500, 33 (2003).

[2] M. Cornacchia et al., Linac Coherent Light Source Design Study Report No. SLAC-R-521, 1998.

[3] S. G. Anderson et al., Phys. Rev. Lett. 91, 074803 (2003).

[4] T. Nakazato et al., Phys. Rev. Lett. 63, 1245 (1989).

[5] S. Heifets and G. Stupakov, Phys. Rev. ST Accel. Beams 6, 064401 (2003).

[6] S. Reiche and J.B. Rosenzweig, Phys. Rev. ST Accel. Beams 6, 040702 (2003).

[7] F. Wang et al., Phys. Rev. Lett. 96, 064801 (2006).

[8] G. L. Carr et al., Nature (London) 420, 153 (2002).

[9] H. Braun et al., Phys. Rev. Lett. 84, 658 (2000).

[10] A.-S. Muller et al., in Proceedings of the 2005 Particle Accelerator Conference, Knoxville (IEEE, Piscataway, NJ, 2005), Vol. 507, p. 2518.

[11] O. V. Chubar and N. V. Smolyakov, J. Opt. (Paris) 24, 117 (1993).

[12] H. Loos et al., in Proceedings of the 2007 Particle Accelerator Conference, Albuquerque, New Mexico, 2007 (IEEE, Albuquerque, New Mexico, 2007), p. 4189. 
[13] K. Brown and R. Servranckx, Report No. SLAC-PUB 3381, 1984.

[14] E. Colby, Ph.D. thesis, UCLA Department of Physics, 1997.

[15] M. Borland, Advanced Photon Source LS-287, 2000.

[16] L. Giannessi and M. Quattromini, Phys. Rev. ST Accel. Beams 6, 120101 (2003).

[17] F. Zhou et al., Phys. Rev. ST Accel. Beams 9, 114201 (2006).

[18] T. Limberg, Ph. Piot, and E. A. Schneidmiller, Nucl. Instrum. Methods Phys. Res., Sect. A 475, 353 (2001).

[19] T. Shaftan and Z. Huang, Phys. Rev. ST Accel. Beams 7, 080702 (2004).
[20] U. Happek et al., Phys. Rev. Lett. 67, 2962 (1991).

[21] R. Lai and A.J. Sievers, Phys. Rev. E 50, R3342 (1994).

[22] P. Muggli et al., Phys. Rev. Lett. 101, 054801 (2008).

[23] D. Schiller et al., Proceedings of the 2007 Particle Accelerator Conference, Albuquerque, New Mexico, 2007 (Ref. [12]), p. 3612.

[24] R. A. Bosch, Nuovo Cimento D 20, 483 (1998).

[25] Y. Shibata et al., Phys. Rev. E 49, 785 (1994).

[26] T. Agoh and K. Yokoya, Phys. Rev. ST Accel. Beams 7, 054403 (2004).

[27] R. Akre et al., Phys. Rev. ST Accel. Beams 11, 030703 (2008). 